\title{
A demonstration of incubation in anagram problem solving
}

\author{
WILLIAM P. GOLDMAN, NIGEL C. W. WOLTERS, and EUGENE WINOGRAD \\ Emory University, Atlanta, Georgia
}

\begin{abstract}
The present study attempted to show that longer periods of incubation would lead to an increased facilitation of anagram problem solving. The break between the presentation of anagrams was $0 \mathrm{sec}, 20 \mathrm{~min}$, or $24 \mathrm{~h}$. It was found that longer breaks increased the probability that an anagram would be solved. These results suggest that solving anagrams may be a useful task for pursuing studies of incubation.
\end{abstract}

Incubation remains a phenomenon resistant to capture in the laboratory. In a trenchant review, Olton (1979) discusses whether the problem is that the laboratory is not a congenial setting for its appearance or that, in spite of anecdotal evidence, incubation is a cognitive illusion. More recently, Browne and Cruse (1988) concluded that what looks like incubation in the laboratory may, in fact, be the outcome of covert rehearsal. The present research represents yet another attempt to demonstrate incubation in the laboratory and was stimulated by our coming across a rarely cited study by Peterson (1974) showing a positive outcome. The task Peterson employed was solving anagrams. Essentially, he found that distributed practice on anagrams yielded more solutions than did massed practice. Although Peterson did not systematically manipulate the interval between successive reappearances of the same word problem, the distributed-practice condition clearly benefited from subjects' leaving the problems and returning to them. It is generally agreed that facilitation of performance attributable to laying a problem aside and returning to it later is a necessary condition for demonstrating incubation. Olton's definition of incubation is pertinent here: "Incubation is a facilitation of thinking that is evident after a period during which no conscious work was done on the task (assuming an earlier period of substantial conscious work)"' (1979, p. 10).

Anagrams have some desirable properties for the study of problem solving. First, they have been widely used and therefore do not represent a totally new research problem. Second, there is a large pool of material to work with, permitting reliable measurement; in many studies, the allor-none solution of a single problem is assessed. Third, the amount of time needed to work on an anagram of a common five-letter word is reasonable, so that one can

This paper was presented at the 35th Annual Meeting of the Southeastern Psychological Association in Washington, DC, March 1989. We thank Jennifer Sykes for assistance in testing subjects. Correspondence concerning this article may be addressed to Eugene Winograd, 532 Kilgo Circle, Department of Psychology, Emory University, Atlanta, GA 30322. comfortably manipulate temporal factors within the boundaries of an experimental session or two.

The present research presents the results of an experiment in which participants tried to solve anagrams. When anagrams were not solved within the time allotted, the subjects returned to them after different periods of time. An incubation-like process can only be considered if solution probabilities for initially failed anagrams are shown to increase with increasing delays between their initial and final presentations.

\section{METHOD}

\section{Subjects}

Seventy-two Emory undergraduates served as participants to fulfill a course requirement.

\section{Materials}

Forty-eight anagrams that had a solution probability between $17 \%$ and $50 \%$ during a 20-sec test period were taken from a study by Polster and Winograd (1989). The solution words were all concrete, highfrequency, five-letter words. Additional anagrams used as filler were made up from the 5,000 most frequent words in the Thorndike and Lorge (1944) word count.

\section{Procedure}

In a between-subject design, three groups of 24 subjects were presented with anagrams and retested at intervals of $0 \mathrm{sec}, 20 \mathrm{~min}$, or $24 \mathrm{~h}$. In the 0 -sec condition, the subjects attempted to solve 48 anagrams that were presented for up to $50 \mathrm{sec}$ each. In the 20-min and 24-h conditions, subjects worked on 36 anagrams for up to $15 \mathrm{sec}$ each and then spent 20 min answering general knowledge questions taken from Yaniv and Meyer (1987). For these two delay conditions, subjects were presented with each unsolved anagram plus 12 new anagrams following the delay. Each of these anagrams was presented for up to $35 \mathrm{sec}$ each. The maximum time allowed for any anagram was $50 \mathrm{sec}$, whether in two segments of 15 and $35 \mathrm{sec}$, as in the two delay conditions, or in one presentation of $50 \mathrm{sec}$, as in the 0 -sec condition. The 12 new anagrams were used to examine general practice effects. Whether an anagram was new or old was counterbalanced across subjects in each condition. Subjects tested $24 \mathrm{~h}$ later were not told about the nature of the second session.

\section{RESULTS}

The proportions of anagrams solved are shown in Table 1 . The top row shows the proportions solved on the 
Table 1

Mean Proportion of Anagrams Solved as a Function of Incubation Period

\begin{tabular}{|c|c|c|c|c|c|c|}
\hline \multirow[b]{3}{*}{ Presentation } & \multicolumn{6}{|c|}{ Incubation Period } \\
\hline & \multicolumn{2}{|c|}{$0 \mathrm{sec}$} & \multicolumn{2}{|c|}{$20 \mathrm{~min}$} & \multicolumn{2}{|c|}{$24 \mathrm{~h}$} \\
\hline & $M$ & $S D$ & $M$ & $S D$ & $M$ & $S D$ \\
\hline 1 & 0.32 & .17 & 0.36 & .16 & 0.34 & .14 \\
\hline 2 & 0.38 & .11 & 0.44 & .15 & 0.49 & .17 \\
\hline
\end{tabular}

Note $-n=24$.

first presentation as a function of the incubation interval. These proportions should be the same across conditions since the groups have not been treated differently on the first presentation. The means are nearly identical and do not differ statistically. The data of interest are shown in the bottom row; these are the proportions of anagrams not solved during the first presentation that were solved on the second presentation. This is the measure of incubation. For the 0 -sec condition, these are the anagrams solved during the last $35 \mathrm{sec}$ of the $50 \mathrm{sec}$ available. The proportion of anagrams solved on the second presentation increases significantly with longer intervals $[F(2,69)$ $=3.48, p<.05]$. A Tukey post hoc analysis shows a significant difference between the 0 -sec and 24 -h conditions $(p<.05)$; none of the other pairwise comparisons was significant.

It is possible, however, that the incubation effect found is not item specific, but instead reflects a general improvement in solving anagrams. Analysis of the new items is relevant to this issue. Each group was presented with 12 new anagrams following the delay; any generalized improvement in solving anagrams would be reflected in performance on these problems. The mean proportions of new anagrams solved within $15 \mathrm{sec}$ were $.32, .36$, and .43 for the 0-sec, 20-min, and 24-h delay conditions, respectively. While these proportions parallel the incubation proportions shown in Table 1 , in this case the outcome is not statistically significant. Still, the trend suggests generalized improvement in solving new anagrams. Therefore, another analysis was conducted to look for stronger evidence for item-specific improvement.
To address this question, an additional analysis was carried out on the anagrams that were repeated after a delay, that is, the failed anagrams in the 20-min and 24-h conditions. We started with the assumption that both failed and new items present during the second session should benefit equally from any source of general improvement such as rest or practice. We then compared the old and new items as follows. Items failed on their first presentation have been worked on for $15 \mathrm{sec}$. What is the proportion of new anagrams not solved during the first $15 \mathrm{sec}$ of work that are solved subsequently? To obtain this value, we looked at the proportion of new anagrams solved after $15 \mathrm{sec}$ of their 35 -sec presentation, that is, new anagrams solved between 15 and $35 \mathrm{sec}$ of work (shown as the broken line in the bottom row of Figure 1).

To make the value for failed, or incubated, anagrams comparable, we examined the proportion of these anagrams solved during the first $20 \mathrm{sec}$ of work during the second presentation (see the broken line in the top row of Figure 1). This equates the incubated and new anagram problems for the amount of solution time available. In brief, we are comparing both sets of anagrams during the period between 20 and $35 \mathrm{sec}$ of attempted solution, omitting those that have been solved during the first $15 \mathrm{sec}$. The only difference is that for the incubated anagrams, there was an interval of either $20 \mathrm{~min}$ or $24 \mathrm{~h}$ intervening between the 15 th and 16 th sec. For the new anagrams, there was no such delay. Table 2 shows the results of this analysis. There were significantly more solutions for the incubated anagrams than for the new ones $[F(1,46)=$ $4.75, p<.05$ ], suggesting that the interval fostered itemspecific improvement. Of course, it is possible that subtle item-selection effects have been overlooked in presenting this analysis.

\section{DISCUSSION}

The present research shows that an incubation-like effect can be obtained with anagrams, thereby supporting the earlier work of Peterson (1974). The essential finding, stripped of all theory, reduces to this: One is more likely to solve a difficult anagram if work on it is interrupted and resumed later than if one spends the same amount of time working

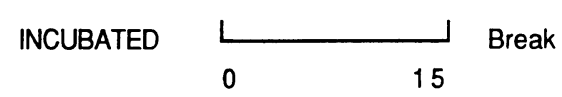

NEW

\begin{tabular}{lll}
\hline 15 & 35 & \\
& & \\
\hline & & \\
\hline 0 & 15 & 15
\end{tabular}


Table 2

Mean Proportion of Anagrams Solved between 15 and 35 Sec as a Function of Incubation Period

\begin{tabular}{lccccc}
\hline & \multicolumn{4}{c}{ Incubation Period } \\
\cline { 2 - 5 } Type of & \multicolumn{2}{c}{$20 \mathrm{~min}$} & & $24 \mathrm{~h}$ \\
\cline { 2 - 6 } \cline { 5 - 6 } Anagram & $M$ & $S D$ & & $M$ & $S D$ \\
\hline Incubated & 0.31 & .10 & & 0.37 & .17 \\
New & 0.27 & .18 & & 0.28 & .20 \\
\hline
\end{tabular}

Note- $n=24$.

on it without interruption. Among the processes that have been suggested as underlying incubation are (1) covert rehearsal, in the sense of active work on the problem during the interval; (2) facilitating cues encountered during the interval; (3) forgetting of inappropriate strategies during the interval (see Smith \& Blankenship, 1989, for a recent demonstration of this factor); (4) reduction of fatigue or rest; and (5) unconscious thought. We agree with Woodworth that the hypothesis that incubation reflects unconscious cognitive processes "should be left as a residual hypothesis for adoption only if other, more testable hypotheses break down"' (1938, p. 823). The research reported here was not designed to test among these hypotheses. Rather, it was intended to see if the basic phenomenon of incubation could be found with anagrams so that more analytic research could be done in the future. In this regard, the results are promising.

Finally, these results raise the possibility that when improvement is shown on a problem after a period of time away from it, improvement may occur at a more general level than that of the specific problem worked on. In future studies of incubation, generalized practice effects, wherein improvement on the entire class of problems occurs, need to be considered as well as problem-specific improvement. Studies using many problems sharing common properties, such as anagrams, are well suited to discriminating between these two kinds of improvement.

\section{REFERENCES}

Browne, B. A., \& Cruse, D. F. (1988). The incubation effect: Illusion or illumination? Human Performance, 1, 177-185.

Olton, R. M. (1979). Experimental studies of incubation: Searching for the elusive. Journal of Creative Behavior, 13, 9-22.

Peterson, C. (1974). Incubation effects in anagram solution. Bulletin of the Psychonomic Society, 3, 29-30.

Polster, M. R., \& WinOgRAD, E. (1989). No evidence of test priming between solving anagrams and completing word fragments. Bulletin of the Psychonomic Society, 27, 303-306.

SMITH, S. M., \& BLANKENSHIP, S. E. (1989). Incubation effects. Bulletin of the Psychonomic Society, 27, 311-314.

THORNDIKE, E. L., \& LORGE, I. (1944). The teacher's word book of 30,000 words. New York: Teachers College Press, Columbia University.

WOODWORTH, R. S. (1938). Experimental psychology. New York: Holt.

YANIV, I., \& MEYER, D. E. (1987). Activation and metacognition of inaccessible stored information: Potential bases for incubation effects in problem solving. Journal of Experimental Psychology: Learning, Memory, \& Cognition, 13, 187-205.

(Manuscript received July 15, 1991.) 